\title{
Die Verwaltung der unternehmerischen Stadt
}

(K)ein Thema in der geographischen Stadtforschung?!

\author{
Felix Silomon-Pflug
}

\begin{abstract}
In der geographischen Stadtforschung finden sich allgemeine Verweise darauf, dass zum Kanon neoliberaler Reskalierung und urbaner Transformation, auch die Einführung von New Public Management in den Städten westlicher Industriestaaten zählt. Daran anschließend argumentiere ich, dass das, was ich als die Verwaltung der unternehmerischen Stadt zusammenfasse, nicht lediglich das Ergebnis abstrakter Neoliberalisierungsprozesse oder technokratischer Modernisierung eines mechanischen Exekutivapparats darstellt. In dem Beitrag zeige ich auf, dass die betriebswirtschaftlich reformierte Verwaltung Effekt und wichtiges Terrain der Ausarbeitung, Artikulation und Durchsetzung einer unternehmerischen Stadtpolitik ist. Dafür schlage ich vor, den Untersuchungsgegenstand städtische Verwaltung als strategisches Feld zu begreifen, das Ergebnis und Terrain gesellschaftlicher Kräfteverhältnisse ist. Des Weiteren begreife ich in den Prozess der Verwaltungsreform als Macht/Wissen-Komplex, um aufzuzeigen, wie sich mit Hilfe von Problematisierungen und Wissensordnungen ein unternehmerisches Verständnis lokalstaatlichen Handelns etabliert. Am Beispiel der Auswirkungen der Verwaltungsreformen auf die Wahrnehmung von und den Umgang mit öffentlichem Liegenschaftsvermögen in Frankfurt/Main und Berlin seit den 1990er Jahren zeige ich zum einen auf, wie über die Dynamisierung und Flexibilisierung der administrativen Apparate sowohl die internen administrativen Strukturen ökonomisiert wurden. Zum anderen stelle ich dar, inwieweit zeiträumlich beschränkte Handlungsformen genutzt wurden und werden, um aufwertungsorientierte Strategien im Kontext einer unternehmerischen Stadtpolitik zu verfolgen.
\end{abstract}

Ersteinreichung: 7. Februar 2018; Veröffentlichung online: 28. November 2018

An english abstract can be found at the end of the document.

\section{Einleitung}

Die Stadt Frankfurt gründet 1995 gemeinsam mit einem Architektur- und Ingenieurbüro eine öffentlich-private Gesellschaft, mit deren Hilfe brachgefallene Liegenschaften des US-Militärs zu attraktiven Wohnquartieren primär für Mittelschichtshaushalte entwickelt werden. Das Projekt wird innerhalb des Magistrats als so erfolgreich bewertet, dass die Gesellschaft - anders als ursprünglich geplant - nicht abgewickelt wird, sondern bis heute als außeradministratives Instrument der Stadtentwicklung genutzt wird. Das Land Berlin beschließt Ende der 1990er Jahre die ertragsorientierte Privatisierung öffentlichen Liegenschaftsvermögens zum Zweck der Haushaltskonsolidierung. Ab 2001 privatisiert eine Tochtergesellschaft des Landes bezirks- und landeseigene Liegenschaften. Über ein disziplinierend-aktivierendes Regime 
unternehmerischer Verwaltungsreformen auf Ebene der Bezirke wird ein beständiger Fluss an verwertbaren Liegenschaften gewährleistet. Ab 2009 stößt diese austeritätspolitische Dominanz im Umgang mit öffentlichen Liegenschaften auf breiten zivilgesellschaftlichen und politischen Widerstand, sodass zumindest die Verwertungsgesellschaft nach mehrjährigen Protest abgewickelt wird.

Beide hier umrissenen Entwicklungen sind Teil einer umfassenden Neuordnung städtischer und kommunaler Verwaltungen, die in den letzten 25 Jahren in Konjunkturen zur Herausbildung dessen geführt haben, was ich als Verwaltung der unternehmerischen Stadt[1] zusammenfasse. Elemente der Verwaltungsreform wie Kostenleistungsrechnung, Produkthaushalte, Zielvereinbarungen oder Budgetierung sind jedoch ebenso wenig als bloße Verfahrensweisen einer veränderten Verwaltungspraxis zu betrachten, wie es sich bei der Gründung privatrechtlicher Gesellschaften durch die öffentliche Hand oder unter deren Beteiligung lediglich um die Verlagerung öffentlicher Aufgaben in eine privatrechtliche Form handelt.

Mit meinem Beitrag möchte ich differenziertes Bild der Rolle ,des Staats ${ }^{\star}$ in urbanen Neoliberalisierungsprozessen zeichnen und aufzeigen, wie die unternehmerische Neuordnung städtischer und kommunaler Verwaltungen die inner- und außeradministrativen Machtbeziehungen bearbeitet und das strategische Feld des lokalen Staats neu konfiguriert. Die Verwaltung der unternehmerischen Stadt begreife ich als institutionell-materiellen Ausdruck der Reskalierung staatlicher und ökonomischer Interventions- und Artikulationsfelder in Form neuer institutioneller Konfigurationen und administrativer Praktiken auf städtischer und regionaler Ebene (vgl. Brenner 2004, Swyngedouw 1997). Bereits Hendrik Lebuhn (2007; 2008; 2010) zeigt in seinen Auseinandersetzungen mit der Einführung von ,New Public Management (NPM) in Berlin, dass Austerität nicht nur als politisches Programm durchgesetzt wird. Privatisierungsdruck und Kosteneinsparungen sind in die administrativen Verfahrensweisen der Berliner Bezirke selbst eingelassen. Besonders anschaulich arbeitet er die Folgen für politische, kulturelle und soziale Initiativen auf, die in besonderem $\mathrm{Maß}$ von den Veränderungen in Folge der Verwaltungsreform betroffen sind. Kritische Auseinandersetzungen finden sich außerdem bei Michael Felder (1998; 2000; 2001), Alessandro Pellizzari (2001; 2004) oder Sünne Andresen et al. (2003). Während Felder sich dezidiert mit den staatstheoretischen Folgen der Einführung von NPM in den deutschen Kommunen beschäftigt, analysiert Pelizzari die Auswirkungen von NPM auf Krankenhäuser in der Schweiz. Andresen et al. beschäftigen sich aus soziologischer Perspektive mit dem Geschlechterwissen und Organisationsverständnis von Akteur_innen der Verwaltungsreform.

Im Folgenden gebe ich zunächst einen kurzen Überblick über die Verwaltungsreform in Deutschland seit den 1990er Jahren. Darauf folgend stelle ich drei Perspektiven auf NPM vor, um daran aufzuzeigen, wie die Neuordnung städtischer und kommunaler Verwaltungen als Gegenstand und Prozess konzeptualisiert werden kann. Hier gehe ich zunächst auf das NPM als Bedingung und Ausdruck neoliberaler Urbanisierungsprozesse ein. Die Neuordnung städtischer und kommunaler Verwaltungen ist nicht nur Effekt urbaner Neoliberalisierungsprozesse, sondern verändert das Terrain 
des lokalen Staates als strategisches Feld zur Ausarbeitung, Artikulation und Durchsetzung einer unternehmerischen Stadtpolitik. Daran anschließend betrachte ich Verwaltung als materiellen Ausdruck gesellschaftlicher Kräfteverhältnisse. Die Reform der lokalen Verwaltungen ist in dieser Perspektive als Reorganisation politischer Herrschaft zu verstehen. Zuletzt betrachte ich den Prozess der Verwaltungsreform als konstruierende Praxis und Macht/Wissen-Komplex. Die diskursive Produktion, Proliferation und Sanktionierung betriebswirtschaftlichen Wissens strukturiert das Handlungsfeld der Kommunen und Verwaltungsmitarbeiter_innen.

Einleitend zu den beiden im Anschluss behandelten Fallbeispielen Frankfurt am Main und Berlin lege ich dar, dass gerade Liegenschaftspolitik und -verwaltung bzw. die Wahrnehmung von und der Umgang mit öffentlichen Liegenschaften einen produktiven Fluchtpunkt für die Auseinandersetzung mit der Neuordnung städtischer und kommunaler Verwaltungen darstellt. Anhand der Fallbeispiele veranschauliche ich, wie sich die Verwaltung der unternehmerischen Stadt in den urbanen Raum einschreibt und diesen als sozialräumliches Gefüge transformiert. Die beiden Fallbeispiele eignen sich, um kontingente Entwicklungspfade der Verwaltungsreform zu veranschaulichen. Während in Frankfurt die binnenadministrative Reform gegenüber privatrechtlichen Gesellschaften eine weniger große Rolle spielt, kam es in Berlin zu einem machtvollen Wirkungszusammenhang beider Reformdimensionen. Die beiden Fallbeispiele unterscheiden sich auch dahingehend, dass in Frankfurt sozio-ökonomische und stadtentwicklungspolitische Interessen des Planungsdezernats im Vordergrund standen, während in Berlin die austeritätspolitischen und haushälterischen Interessen der Senatsverwaltung für Finanzen dominieren. In beiden Fallbeispielen lässt sich anhand der Liegenschaftspolitik und -verwaltung aufzeigen, wie die unternehmerische Verwaltungssteuerung als wirkungsvoller Prozess der Neoliberalisierung fungiert.

\section{Die Reform städtischer und kommunaler Verwaltungen als Krisendiskurs}

Die beiden eingangs skizzierten Entwicklungen sind Teil zahlloser Reforminitiativen, über die seit den frühen 1990er Jahren in Konjunkturen[2] in deutschen Städten und Kommunen Verwaltungsstrukturen und -handeln betriebswirtschaftlich rationalisiert werden. Die Reformbestrebungen in den deutschen Städten und Kommunen werden gemeinhin unter dem Begriff des ,Neuen Steuerungsmodells‘ (NSM) gebündelt. Das NSM stellt das begrifflich-programmatische Pendant zum angelsächsischen NPM dar, ohne jedoch daraus abgeleitet werden zu können. Das NSM - als handlungsleitender Rahmen für die Schaffung einer (international) wettbewerbsfähigen Verwaltung - wird in Deutschland ab den 1990er Jahren von der „Kommunalen Gemeinschaftsstelle für Verwaltungsmodernisierung“ (KGSt) entwickelt und vorangetrieben. Eine weitere bedeutende Akteurin ist dabei die Bertelsmann Stiftung, die bis Ende der 1990er Jahre in enger Zusammenarbeit mit der KGSt die Mobilisierung unternehmerischer Verwaltungsreformen vorantreibt (vgl. Bertelsmann Stiftung 1994a; 1994b, Adamaschek 1997). Daneben sind es Verwaltungswissenschaftler_innen, 
partei- und gewerkschaftsnahe Stiftungen, kommunale Interessensverbände und Vertreter_innen aus der Verwaltungspraxis, die die Verwaltungsreform ab den frühen 1990er Jahren begründen und mobilisieren. Ab Mitte der 1990er Jahre entdecken zudem namhafte Beratungsunternehmen ,Public Management‘ als neues Geschäftsfeld.

Der über verwaltungswissenschaftliche und administrative Fachpublikationen sowie in Gutachten, Berichten und Reports mobilisierte Reformdiskurs des NSM bildet eine dialektische Einheit aus Problematisierungen einerseits sowie Lösungsstrategien und -instrumenten anderseits. Im Krisendiskurs des NSM wird das Problem der Verwaltung primär als technisches und verwaltungsimmanentes Problem formuliert: Eine Verwaltung, „deren Aufgabe vorwiegend darin besteht, das geltende Recht korrekt anzuwenden, steht uns heute im Weg [...] [und] kann daher keinen modernen Leistungsbegriff entwickeln“(Banner 1994: 352). Dieser Krisendiskurs über die Dysfunktionalität kommunaler Verwaltungen zeichnet sich durch eine gewisse Körperlichkeit aus. So wird beispielsweise eine „Naturwüchsigkeit‘ der Ausweitung staatlicher Aufgaben“ (Naschold 1995: 71) attestiert oder in einer Publikation des Deutschen Beamtenbundes (1996: 13) dazu festgestellt: „Allseits formiert sich Kritik [...] gegen wasserköpfige Verwaltung, gegen aufgeblähten, überteuerten öffentlichen Dienst.“

Das Bild einer adipösen, zur Expansion neigenden und leistungsunfähigen Verwaltung repräsentiert eine verwaltungsimmanente Krise. Dabei bleiben Fragen nach Steuerpolitik und (Um-)Verteilung des gesellschaftlichen Reichtums, nach Globalisierung und ökonomischem Strukturwandel oder Fragen nach direkter Mitbestimmung und Partizipation im Diskurs ausgeblendet. Mit dem Krisendiskurs ist eine Komplexitätsreduzierung und politische Imagination verbunden, die in einer Objektivierung der dysfunktionalen Verwaltung resultieren (vgl. Jessop 2010). Mit Crispian Fuller (2010) gesprochen markiert der Krisendiskurs nicht notwendigerweise eine strukturelle Krise kommunaler Verwaltungen, sondern ist strategischer Ansatzpunkt, um das institutionelle Gefüge der Verwaltungen als Feld für Reformen und Interventionen zu öffnen. Es sind die handlungsfähigen Kommunen selbst, die - unabhängig von einer Reformgesetzgebung - eigenverantwortlich Reforminitiativen entwickeln und umsetzen sollen und vor allem auch können. In dem Reform- und Krisendiskurs werden städtische und kommunale Verwaltungen als zu Reformierendes und Reformbares konstituiert. Die diskursive Problematisierung der Verwaltung wiederum strukturiert das Handlungsfeld der Reformen und beschränkt es auf die betriebswirtschaftliche Rationalisierung binnenadministrativer Strukturen in Städten und Kommunen.

Dieser Rationalisierungsprozess bedeutet nach Meinung des kommunalen Fachverbands KGSt „die Effektivität, Effizienz, Qualität und Kundennähe kommunaler Dienstleistungen mit Hilfe angepaßter betriebswirtschaftlicher Instrumente und Verfahren aus dem privaten Sektor zu verbessern“ (KGSt 1992: 3). Ziel der Reformen ist nicht primär die Privatisierung staatlicher Leistungen und Aufgaben, es ist vielmehr die Verwaltung selbst, die wie ein (Dienstleistungs-)Unternehmen organisiert wird (vgl. Banner 1991, KGSt 1992; 1993b). Weder die korrekte Anwendung geltenden Rechts noch die Schaffung neuer Verwaltungseinheiten oder die Einstellung von mehr 
Personal werden von den Reformer_innen als adäquate Instrumente zur Krisenbewältigung betrachtet.

Mit der Verwaltungsreform sei es gelungen, das „Konzept des Regierens und Verwaltens auf die veränderten Anforderungen der Globalisierung anzupassen [...]“ (Koch 2004: 2). Allgemeiner stellt die KGSt fest: „Eine leistungsfähige Kommunalverwaltung ist im zusammenwachsenden Europa ein Standortfaktor ersten Ranges“ (KGSt 1992: 3). Diesem Verständnis nach ist eine leistungsfähige und wirtschaftlich handelnde Verwaltung Bedingung, um im interlokalen Wettbewerb um Investitionen, Unternehmensansiedlungen, Arbeitskräfte und Fördergelder erfolgreich zu sein. Im Laufe der 1990er Jahre führt die strategische Multiplizierung und Dezentralisierung der Reforminitiativen zu einer raumzeitlich und inhaltlich fragmentierten Reformlandschaft (vgl. Silomon-Pflug 2018: 127-135). Diese mangelnde Homogenität der multiplen Reformbestrebungen ist jedoch weniger als Scheitern, sondern vielmehr als Normalisierung der Verwaltungsreform im Sinne eines „failing forward“ (Peck 2010: 7) zu betrachten.[3]

\section{Perspektiven auf New Public Management}

\subsection{NPM als Bedingung und Ausdruck neoliberaler Urbanisierung}

In der geographischen Stadtforschung finden sich allgemeine Verweise darauf, dass zum Kanon neoliberaler Transformationen, Re-Strukturierungen und Neuordnungen auch die Einführung von NPM in den Städten westlicher Industriestaaten zu zählen ist:

„[C]ities [...] have become increasingly important geographical targets and institutional laboratories for a variety of neoliberal policy experiments, from place-marketing, enterprise and empowerment zones, local tax abatements, urban development corporations, public-private partnerships, and new forms of local boosterism to workfare policies, property-redevelopment schemes, business-incubator projects, new strategies of social control, policing, and surveillance, and a host of other institutional modifications within the local and regional state apparatus." (Brenner/Theodore 2002: 368, e. H.)

Am Ende der langen Aufzählung an Konkretisierungen, welche Prozesse die Neoliberalisierung des Städtischen auszeichnen, verweisen Brenner und Theodore darauf, dass dazu auch, andere institutionelle Modifikationen innerhalb des lokalen und regionalen Staatsapparats zu zählen sind. Über diese Feststellung hinaus wird jedoch nicht weiter behandelt, wie sich die neoliberale Neuordnung städtischer und kommunaler Verwaltungen artikuliert und welche Folgen sie für das sozialräumliche Gefüge Stadt haben. NPM ist lediglich ein möglicher Ausdruck abstrakter urbaner Neoliberalisierungsprozesse. Diese werden als das Ergebnis staatlicher Reskalierungsprozesse betrachtet (Brenner 2004), und es kommt im Rahmen unternehmerischer Stadtpolitiken zur Herausbildung neuer Formen von urban governance (Harvey 1989). Jennifer Robinson (2011: 31-32) verweist darauf, dass „questions of competitive positioning, image, representation, and strategic planning, as well as to 
classic NPM concerns with civic partnership and efficiency in service delivery" ab den 1990er Jahren zunehmend an Bedeutung gewonnen haben. Eugene McCann und Kevin Ward (2011: xviii) stellen fest, dass „[c]ities and the local state have [...], through innovations like the New Public Management, been reconceived as a facilitative, rather than regulatory, apparatus, behaving like a business to attract and support capital, rather than to promote welfare as understood under Keynesianism“. Susanne Heeg (2008: 45) sieht darin den Wandel „von einer sozial ausgleichenden und umverteilenden Politik zu proaktiven, angebotsorientierten Wirtschaftsstrategien“. Der damit verbundene institutionelle Umbau des lokalen Staates gehe mit Ausgründung und Privatisierung öffentlicher Aufgaben einher.

Die bloße Feststellung reicht jedoch nicht aus, dass auch der politisch-administrative Apparat des lokalen Staats Bedingung und Gegenstand sozialer, politischer und ökonomischer Reskalierungsprozesse ist. Die Frage danach, wie sich diese Neuordnungsprozesse und mit welchen Auswirkungen vollziehen, bleibt unbeantwortet. Nach Erik Swyngedouw (1997: 142) gründen Veränderungen in der Relevanz bedeutender ,scales' oder deren Entstehung auf ,changes in the geometry of social power by strengthening the power and the control of some while disempowering others“. Vor diesem Hintergrund ist auch die Verwaltungsreform in den deutschen Städten und Kommunen zu analysieren, um ein weitergehendes Verständnis davon zu entwickeln, wie und warum sich das Lokale zu einer bedeutenden Maßstabsebene politischer und ökonomischer Intervention herausgebildet hat.

\subsection{NPM als Ausdruck politischer Herrschaft}

Eine sehr umfassende und zugleich limitierte Auseinandersetzung mit der Verwaltungsreform in deutschen Städten und Kommunen findet sich in deren Haus- und Hofdisziplin, den Verwaltungswissenschaften. In unzähligen Publikationen über die Verwaltungsreformen im Allgemeinen und das NSM im Speziellen wird die kommunale Verwaltung auf einen technokratischen Exekutivapparat reduziert. In umfangreichen Studien werden Implementationsbedingungen und -hindernisse, Umsetzungsbreite und -tiefe sowie die (Un-)Wirksamkeit der Reformen innerhalb der gegenständlichen Grenzen untersucht (vgl. etwa Jann et al. 2004, Bogumil et al. 2007). Es ist diese institutionen- und prozessimmanente Betrachtung, die die Erklärungskraft der verwaltungswissenschaftlichen Auseinandersetzungen nicht nur limitiert, sondern auch wesentlich zur Mobilisierung und Stabilisierung der Verwaltungsreformen beiträgt.

Bereits 1982 stellen Josef Esser und Joachim Hirsch fest, dass nicht die „Betriebswirtschaftslehre des Staates“ (Esser/Hirsch 1982: 105) im Zentrum einer Auseinandersetzung mit dem Staat stehen solle, sondern die staatsadministrative Vermittlung und Reproduktion der kapitalistischen Klassenbeziehungen auf dem Terrain des Staates. Diese Prozesse seien nur zu verstehen, wenn „die Bedingungen und Grenzen der Staatstätigkeit, die Formen administrativ-politischer Krisenverarbeitung, die [...] innerapparativen Strukturveränderungen und Prozesse und deren Rückwirkung auf die gesellschaftlichen und politischen Handlungsspielräume" (Esser/ Hirsch 1982: 106) eingehend analysiert werden. Die Perspektive ist 
deutlich von einer deutschen Verwaltungswissenschaft abzugrenzen, die als „Geleitzug“ (Damkowski/Precht 1998: 18-19) der Verwaltungsreform fungiert und deren Kern eine verwaltungsimmanente Bürokratie- und Implementationsforschung bildet, für die „die möglichst große Nähe des Wissens zum Kontext seiner Anwendung als Voraussetzung instrumenteller Verwertbarkeit gilt“" (Vogel 2006: 499).

In Anschluss an Nicos Poulantzas konzeptualisiert Alex Demirović (2007a: 118), ähnlich wie Esser und Hirsch, den Staat als strategisches Feld „auf dem sich die machtblockinternen Widersprüche und Klassenkämpfe entsprechend seinen eigenen Bedingungen in seinem, materiellen Gerüst', seiner ,Dichte' und ,Widerstandskraft' auswirken können“. Weiter argumentiert er, dass auch die Verwaltung im engeren Sinne - beispielsweise die Schaffung und Dauer von Einrichtungen oder die Beschäftigung von Staatspersonal sowie dessen Qualifikation - als Kräfteverhältnis zu verstehen ist (vgl. Demirović 2007a: 120-121). Aus diesem Grund ist die Verwaltung nicht als homogenes, widerspruchsfreies und technisches Institutionenensemble zu begreifen, sondern als zeit-räumlich spezifischer Ausdruck einer „Vielzahl zerstreuter Mikropolitiken“ (Poulantzas 2002: 167).

Das bedeutet, dass der Staat die dynamisch-kontingente Verdichtung gesellschaftlicher Kräfteverhältnisse ist, die ihn hervorbringen, durchziehen und transformieren. Von diesem Verständnis ausgehend spricht Bob Jessop von einer strategischen Selektivität des Staates und seines materiellen Gerüsts. Damit spezifiziert er, „daß bestimmte Staatsformen einige Strategien gegenüber anderen, den Zugang einiger Kräfte gegenüber anderen, einige Interessen gegenüber anderen, einige Zeithorizonte gegenüber anderen, einige Koalitionsmöglichkeiten gegenüber anderen privilegieren“ (Jessop 1992: Fn. 3). Der Staat und seine Apparate verfügen trotz oder mehr noch wegen ihrer fraktionierten und von Machtbeziehungen hervorgebrachten Materialität über eigene Handlungsressourcen und relationale Autonomie gegenüber anderen gesellschaftlichen Sphären.

Die binnenadministrative Rationalisierung ist strategischer Ansatzpunkt für die Rekonfiguration des strategischen Felds des lokalen Staats und damit die Bedingungen der Artikulation, Transformation und Materialisierung von Interessen gegenüber dem Staat und durch ihn: „Die staatlichen Apparate müssen reorganisiert, ihre bisherigen Verflechtungen mit der Ökonomie reartikuliert werden" (Demirović 2007b: 25). Besonders anschaulich wird dies von Dietrich Budäus und Dennis Hilgers dargelegt, wenn sie problematisieren, dass für die diagnostizierte strukturelle Ineffizienz der öffentlichen Verwaltungen auch eine ,gesellschaftspolitisch induzierte personale Komponente für die Erklärung der [...] , organisierten Unverantwortlichkeit" von Bedeutung“ sei. Konkret benennen sie hier „die (versuchte) Instrumentalisierung von Staat und Verwaltungen durch die 68er Generation“ (Budäus/Hilgers 2010: 85-86). Im Zuge des ,Marschs durch die Institutionen' seien Personalentscheidungen nicht nach Qualifikation, sondern nach politischen und ideologischen Kriterien getroffen worden, und es sei zu einer personellen Erweiterung der Verwaltungen gekommen. Dies hätte verhindert, dass in den Verwaltungen auf die sozialen und ökonomischen Veränderungen frühzeitig reagiert wurde. Die Konstatierung der Krise der öffentlichen Verwaltungen und die damit verbundene Dynamisierung, 
Flexibilisierung und Diffusion der lokalen Staatsapparate sind strategische Ansatzpunkte für die Durchsetzung bzw. Sicherstellung politischer Herrschaft: „Die Verwaltung selbst sollte wieder stärker an die bürgerlichen Kräfte angebunden, die Repräsentation ihrer Interessen verstärkt werden“ (Demirović 2011: 86). Dies geschieht durch deren Bearbeitung als strategisches Handlungs- und Möglichkeitsfeld, auf dem gesellschaftliche Konflikte ausgetragen werden und sich materialisieren.

\subsection{NPM als konstruierende Praxis}

Die Neuordnung städtischer und kommunaler Verwaltungen als Macht/ Wissen-Komplex zu begreifen, dient einer weiteren Schärfung der im vorherigen Abschnitt bereits skizzierten Abkehr von der inneren Betriebslogik lokalstaatlicher Verwaltungen und deren Reform. Als Macht/Wissen-Komplex (vgl. Foucault 1977) betrachtet ist die Verwaltungsreform nicht ein technisches Reformprogramm, sondern konstituiert ein Wissensfeld und Set aus Strategien, Praktiken und Technologien, das administratives Handeln und die in ihr tätigen Subjekte als Objekte der Intervention und zu Reformierendes produziert. Die diskursive Kombination aus Problematisierung und Aktivierung im Kontext der Verwaltungsreformen beschreibt nicht lediglich ein administratives System. Die Produktion, Verbreitung und Sanktionierung handlungsleitenden Wissens im Krisen- und Reformdiskurs ist untrennbar verbunden mit der Mobilisierung der Reformen (unter anderem in Form von Gutachten und Berichten, Modellprojekten oder Wettbewerben) und dem unternehmerischen Verwaltungshandeln selbst (beispielsweise Kostenleistungsrechnung, Produkthaushalte, Budgetierung oder Qualitätsmanagement).

Die unternehmerische Verwaltungsreform fungiert als handlungsleitende Wissensordnung, die das Handlungsfeld der Städte und Kommunen strukturiert und gleichzeitig deren Handlungsautonomie in den Vordergrund stellt. Die Reform der öffentlichen Verwaltungen ist eine diskursive Praxis, „die in einem Feld die Begriffe mit Bedeutung füllt, die Objekte bezeichnet, diese damit erst sozial wahrnehmbar macht“ (Diaz-Bone 2006: 73). Es handelt sich um eine konstruierende Praxis, die Wissensordnungen konstituiert und im dialektischen Verhältnis zu gesellschaftlichen Praktiken steht (vgl. Foucault 1981: 231, siehe auch Belina/Dzudzek 2009: 142, Adolphs 2008: 184). Die Verwaltung der unternehmerischen Stadt ist damit Konvergenzpunkt und Verdichtung einer Vielzahl heterogener lokaler und überlokaler Strategien, Praktiken und Technologien der Neoliberalisierung (vgl. Goodwin/Painter 1996: 637, Ong 2006). Das, was als Neoliberalisierung gefasst werden kann, konstituiert sich in der materiellen Praxis der Subjekte und durch diese hindurch.

Die Neuordnung städtischer Verwaltungen - verstanden als Macht/WissenKomplex - umfasst einerseits in einem problematisierenden Reformdiskurs ein neues Verständnis der unternehmerisch handelnden Verwaltung. Andererseits verändert sie davon untrennbar auch die materiellen Praktiken, die das bürokratische Gerüst des lokalen Staats hervorbringen, durchziehen und transformieren. Das Ergebnis ist die Reartikulation inner- und außeradministrativer Strategien und Praktiken. Kurz gesagt konstituiert die Neuordnung städtischer und kommunaler Verwaltungen eine staatstransformierende Praxis: Zur 
Disposition steht sowohl, welche Aufgaben die lokalen Verwaltungsapparate erbringen, als auch, wie diese erbracht werden. Dies gilt jedoch nicht nur für Prozesse der Institutionalisierung, sondern auch der Subjektivierung: „Subjects or actors [...] depend on specific discursive conditions of possibility“ (Gottweis 2004: 253). Es sind eben jene Möglichkeitsbedingungen legitimen Verwaltungshandelns, die mit der Verwaltungsreform in den deutschen Städten und Kommunen verändert werden.

Davon ausgehend ist es unmöglich, Verwaltungen als Gegenstand und Verwaltungsreformen als Prozess als einander äußerlich zu begreifen. Vielmehr rücken Praktiken, Strategien und Technologien in den Fokus, deren Effekt die Verwaltung der unternehmerischen Stadt ist. Es handelt es sich um einen Prozess, bei dem „the mechanisms of government themselves become the subject of problematization and scrutiny" (Gottweis 2004: 263). Die zugrundeliegende Reformrationalität zeichnet sich nicht durch ein bestimmtes Reformziel aus, sondern durch eine beständige Befragung und Infragestellung von Verwaltungsstrukturen und -handeln, die in Form einer betriebswirtschaftlichen Überprüfung stattfindet. In der Logik der Verwaltungsreformen steht Verwaltungshandeln immer unter dem Verdacht des Übermaßes. Mit Hilfe unternehmerischer Verwaltungssteuerung soll sich Verwaltungshandeln entlang betriebswirtschaftlichen Wissens auf ein vermeintlich objektives Maß selbst beschränken.

\section{Liegenschaftspolitik und -verwaltung in der unternehmerischen Stadt}

Für die Auseinandersetzung mit der Neuordnung städtischer und kommunaler Verwaltungen eignen sich Liegenschaftspolitik und -verwaltung als Fluchtpunkt, um die Auswirkungen der Reformen auf die Geographie des urbanen Raums zu untersuchen.[4] In einem in Berlin geführten Interview wird diesbezüglich festgestellt, „dass sich an den Liegenschaften was fokussiert: Die öffentliche Hand hat nichts, aber sie hat Grundstücke. Und die Frage ist, weil die Grundstücke nicht hergestellt sind, sondern zur Substanz der Gemeinde gehören, wie gehen wir eigentlich damit um?"(B2012-B: 245) Ähnlich formulierte dies bereits Karl Polanyi (vgl. 2001: 71-80), wenn er feststellt, dass Land historisch schon immer zentraler Gegenstand von Regulation war. Für ihn bedeutet Land - wie auch Arbeit und Geld - als fiktive Ware unter Marktmechanismen zu handeln, „to subordinate the substance of society itself to the laws of the market“ (2001: 75).

Darüber hinaus ist die Frage öffentlicher Daseinsvorsorge - beispielsweise Schul- und Sozialpolitik, Gesundheit, Kultur, Verkehr oder Wirtschaftsförderung und Stadtentwicklung - eng verknüpft mit eben jener Frage nach der Wahrnehmung von und dem Umgang mit (öffentlichem) Liegenschaftseigentum, die mit der Neuordnung städtischer und kommunaler Verwaltungen neu gestellt wird. Unter welchen Bedingungen, von wem und mit welchen Zielen Liegenschaften der öffentlichen Hand genutzt werden können, ist damit gerade in hochverdichteten urbanen Räumen Gegenstand von Interessens- und Regulationskonflikten: Erstens sind öffentliche Liegenschaften ein Kostenpunkt im Haushalt einer Kommune. Die liegenschaftsbezogenen Kosten können durch Aufgabe, Umnutzung, 
Modernisierung oder marktorientierte Bewirtschaftung reduziert werden. Zweitens ist Liegenschaftsvermögen auch eine Handlungsressource, die es zum Beispiel ermöglicht, Bildungseinrichtungen zu bauen, Wohnraum zu schaffen oder Unternehmen anzusiedeln.[5] Im Rahmen austeritätspolitischer Maßnahmen sind Liegenschaften nicht zuletzt eine Vermögensressource, deren Aktivierung in Form von privaten Investitionen oder Privatisierung die Generierung finanzieller Gewinne ermöglicht.

In den folgenden beiden Abschnitten wird anhand der beiden Fallbeispiele Frankfurt am Main und Berlin dargestellt, wie die betriebswirtschaftliche Neuordnung der städtischen und kommunalen Verwaltungen die Wahrnehmung von und den Umgang mit öffentlichem Liegenschaftsvermögen transformiert haben. Es dominiert ein Verständnis öffentlichen Eigentums, das gegenüber privatem Eigentum nur „provisional acceptance [...] in certain situations" (Blomley 2003: 7) oder in Form von Infrastruktur genießt. Anhand der beiden Fallbeispiele lässt sich das Argument veranschaulichen, dass die Verwaltung der unternehmerischen Stadt die Bedingungen und Formen der Interessensartikulation gegenüber dem Staat und durch ihn in limitierender Art und Weise transformiert und institutionelle Handlungsräume einer unternehmerischen Stadtpolitik schafft bzw. strukturiert.

\subsection{Frankfurt am Main: Private Gesellschaften als Verwaltungsbypässe}

In Frankfurt am Main stellt sich ab 1992 mit dem Abzug des US-amerikanischen Militärs die Frage der Umnutzung der brachgefallenen Areale, die zunächst in das Eigentum des Bundes fielen. Von einem an der Entwicklung der Militärliegenschaften beteiligten Stadtplaner wird die Haltung der damaligen Stadtregierung gegenüber den freiwerdenden Flächen folgendermaßen zusammengefasst: „Da hält man sich am besten raus. Da macht man mal die Bebauungspläne und dann wird der Markt das schon machen." (F2014-U: 45)[6] Jedoch wurden die Areale am freien Grundstücks- und Immobilienmarkt nicht nachgefragt. Neben der Größe und der unattraktiven Lage waren auch mögliche Altlasten ein Grund dafür. Gleichzeitig stellte sich in den 1990er Jahren in Frankfurt das Problem, dass die Stadt Auflagen der kommunalen Aufsichtsbehörden zur Konsolidierung des städtischen Haushalts zu erfüllen hatte. Mit knapp 3,5 Milliarden Euro erreichte der Schuldenstand der Stadt 1992 seinen historischen Höchststand.

Dementsprechend heißt es im Vortrag des Magistrats an die Stadtverordnetenversammlung zur Gründung der öffentlich-privaten Partnerschaft (ÖPP) mit einem Architektur- und Ingenieurbüro zur Entwicklung zweier großer Kasernenareale: „Die begrenzten Finanzmittel [...] erschweren die Möglichkeit, diese Flächen aus eigenen städtischen Mitteln vom Bund zu erwerben, zu entwickeln und anschließend zu vermarkten." (Koenigs/ Wentz 1994: 1) Es ist jedoch nicht nur die Sparpolitik, weswegen die Areale nicht von der Stadt selbst erworben wurden, wie von einem Mitglied der Frankfurter Stadtverordnetenversammlung beschrieben wird:

„Es ist eigentlich unumstritten, dass man solche Flächen nur mit einer GmbH-Struktur entwickeln kann [...]. Mit so einer GmbH [...] haben Sie 
eine schlagkräftige Struktur, die auch flexibel ist [...]. [D]ie städtischen Strukturen in der Verwaltung, [...] die ganze Genehmigungsstruktur mit Kämmerei, Revisionsamt, Personal- und Organisationsamt und teilweise mit Genehmigung durch die Kommunalaufsicht [...] ist halt eine sehr schwerfällige Struktur, die für eine wirtschaftliche Entwicklung von solchen Gebieten unangemessen ist.“ (F2013-T: 81)

Die mangelnde Wirtschaftlichkeit wird als Ausdruck mangelnder Leistungsfähigkeit der öffentlichen Verwaltung angesehen und dient der Legitimation neuer institutioneller Handlungsräume. Vor diesem Hintergrund ist die Gründung der Gesellschaft als institutionell begrenzte und projektbezogene Umsetzung der Ziele des NSM zu sehen, ohne dass dafür in den städtischen Gremien eine umfassende Reforminitiative in der Gesamtverwaltung diskutiert und umgesetzt werden musste.[7] Hinzu kommt, dass die ÖPP-Gesellschaft kein eigenes Personal hat, die private Gesellschafterin mit der Geschäftsbesorgung beauftragt ist und die amtierende Stadtplanungsdezernent_in den Aufsichtsratsvorsitz innehat. So betrachtet handelt es sich um eine rechtliche Hülle bzw. Briefkastenfirma. Diese wird als flexibles Instrument genutzt, um unabhängig von den öffentlichen Verwaltungs-, Mitbestimmungs- und Finanzierungsstrukturen die gewünschten Handlungs- und Finanzierungressourcen zu erschließen, um die Brachflächen,wirtschaftlich` zu entwickeln - was bedeutet, dass private Geldgeber_innen ihre Gewinnerwartungen realisieren können.

Mit der ÖPP selbst sind jedoch keine unmittelbaren finanziellen Zielsetzungen verbunden. Bis auf eine Ausschüttung in Höhe der Eigenkapitaleinlage werden keine Gewinne erwirtschaftet, die an die beiden Gesellschafter_innen ausgezahlt würden. Sie dient vielmehr als Instrument der Problemlösung, wobei das Problem darin besteht, dass Liegenschaften nicht vom Markt angenommen werden, aber ein politisches Interesse besteht, diese einer (aufwertenden) Nutzung zuzuführen: „Wenn [...] der normale Marktteilnehmer sagt: Lohnt sich nicht, kann man nicht ordentlich Geld verdienen. Dann stellt sich die Frage: Wäre das nicht was für diese Gesellschaft?“ (F2013-U: 19)

Mit dem ÖPP wurde vom Planungsdezernat gemeinsam mit dem ausführenden Planungsbüro die institutionelle Struktur außerhalb der bestehenden Verwaltung geschaffen, um eine unternehmerische Stadtpolitik umzusetzen. Der damalige Planungsdezernent Martin Wentz (SPD) stellt bereits 1991 fest, dass sich „gesellschaftliche Gruppen wie die neuen ungebundenen Mittelschichten [...] zu einem sozial und politisch relevanten Faktor innerhalb der städtischen Bevölkerung entwickelt“ haben (1991: 11-12). In einem Interview mit einem Stadtplaner wird die Situation in Frankfurt in den frühen 1990er Jahren folgendermaßen problematisiert: „Wenn Sie eine ganze Bevölkerungsgruppe so behandeln, dass Sie überhaupt kein Angebot für die in der Stadt haben, ist es ja eine Katastrophe." (F2013-U: 49)

Es ist vor allem diese Orientierung an neuen urbanen Mittelschichten, die als ,soziale Rendite“ mit der Inwertsetzung der Brachflächen als politisches Ziel verbunden ist. Zur Umsetzung dieses Ziels wurde mit der ÖPP ein Bypass um die administrativen Strukturen, städtischen Mitbestimmungsprozesse und Fragen der Finanzierung geschaffen. Die Gründung der 
Konversionsgesellschaft verändert das strategische Terrain des lokalen Staats dahingehend, dass von Seiten der Stadt selektive Schnittstellen geschaffen wurden, um nicht markgängige Brachflächen im öffentlichen Eigentum für kapitalstarke Immobilienentwickler attraktiv und zugänglich zu machen. Die Gesellschaft stellt die institutionelle Form dar, in der eine öffentliche und private unternehmerische Stadtpolitik in Dialog gebracht und synchronisiert werden.

Das ÖPP zur Brachflächenvalorisierung ist jedoch nur eine Spielart von ähnlichen Gesellschaften, mit denen in Frankfurt städtebauliche und stadtentwicklungspolitische Vorhaben realisiert wurden. Konversionsprojekte wie das Deutschherrnufer auf dem ehemaligen Schlachthofareal, die Konversion des Westhafens von einem Gewerbehafen in ein hochpreisiges Wohn- und Arbeitsquartier oder die Konversion des Rebstockareals sind weitere Beispiele, bei denen private Gesellschaften von der Stadt Frankfurt genutzt wurden, um vormals gewerblich und industriell genutzte Brachen mit privatem Geld und Knowhow zu entwickeln. Dieses Vorgehen ermöglicht die Finanzierung und (politische) Durchsetzung einer unternehmerischen Stadtpolitik in einer marktkompatiblen Form und jenseits des traditionellen administrativen Terrains beziehungsweise der Mitbestimmungsstrukturen, die die wohnungspolitische Situation in Frankfurt bis heute prägt (vgl. Schipper 2018).

\subsection{Berlin: Ausverkauf des öffentlichen Liegenschaftsvermögens}

Ab dem Jahr 2001 beginnt eine Gesellschaft des Landes Berlin öffentliches Liegenschaftsvermögen zu privatisieren, um mit den Privatisierungsgewinnen einen Beitrag zur Konsolidierung des Landeshaushalts zu erzielen. Die Privatisierung von Liegenschaften beginnt jedoch nicht erst mit dem neu geschaffenen Liegenschaftsfonds. DieZuständigkeit lag zuvor bei den Berliner Bezirken und stellt deren einzige Möglichkeit dar, eigene Einnahmen zu generieren.[8] Mit seiner Gründung fungiert der Fonds als zentraler Ansprechpartner und beginnt die aktive Vermarktung seines Portfolios beispielsweise auf internationalen Immobilienmessen, um Investor_innen anzusprechen.

In den zehn Jahren nach seiner Gründung privatisiert die Gesellschaft Liegenschaftsvermögen im Umfang von 16 Millionen Quadratmetern und erzielt dabei Erlöse in Höhe von circa 2,4 Milliarden Euro, die in erster Linie zur Reduzierung der Zinslast genutzt werden. Neben werthaltigen Liegenschaften, die vor allem für Investor_innen von Interesse sind, privatisierte der Fonds auch Baugrundstücke für Ein- und Zweifamilienhäuser. In beiden Fällen dominiert das sogenannte Bieter_innenverfahren. In einer Broschüre zum Verkauf von Baugrundstücken für Ein- und Zweifamilienhäuser heißt es zu dem Verfahren: „Einen Preis suchen Sie hier vergeblich, denn Sie bestimmen, was Ihnen ,Ihr` Grundstück wert ist!“ (Liegenschaftsfonds Berlin 2014) Diese Orientierung am sogenannten Potentialwert einer Liegenschaft führt dazu, dass der Fonds und damit das Land Berlin selbst davon profitieren beziehungsweise daran interessiert sind, dass die Liegenschaften von den Käufer_ innen möglichst hochwertig entwickelt werden. In diesem Zusammenhang beriet der Fonds in Einzelfällen Berliner Bezirke, um ein verkaufsförderndes Planungsrecht zu schaffen (vgl. Silomon-Pflug 2018: 197-199). 
Die Schaffung einer privaten Gesellschaft wird von einem ehemaligen Staatssekretär auch damit begründet, „dass man professionelle Arbeit nur außerhalb der Verwaltung bekommt“. Dies sei „,vor allen Dingen ideologisch geprägt, [...] aber natürlich auch von Erfahrungen, dass jede Verwaltung ihre eigenen Interessen hat." (B2012-A: 123) Oder pointierter von einem Mitglied des Berliner Abgeordnetenhauses: „Ein derartiger Verkauf [...] wäre ja völlig unmöglich gewesen aus der Verwaltung heraus. Undenkbar!“(B2012-H: 65) In Abgrenzung gegenüber den Verwaltungsmitarbeiter_innen wird der Fonds als Instrument gesehen, Tarifflucht nach oben zu begehen. Auf diese Weise war es möglich, unabhängig von den tariflichen Bestimmungen des öffentlichen Diensts immobilienwirtschaftlich qualifiziertes Personal einzustellen.

Es kommt im Ergebnis zu einer grundlegenden Verschiebung in der Wahrnehmung öffentlichen Liegenschaftsvermögens als aktivierbare Vermögensressource.[9] Wie Lebuhn (2010: 40) feststellt, wird mit der Einführung des NSM in den Bezirken „ein Verwertungsdruck erzeugt, der ungenutzte öffentliche Liegenschaften einem unmittelbaren Privatisierungsdruck unterwirft.“ Dazu trägt bei, dass es mit der Umstellung auf eine unternehmensähnliche Haushaltsführung auf Ebene der Bezirke zur Reformulierung aller Verwaltungsleistungen in Form von Produkten und der Erfassung aller produktspezifischen Kosten kommt. Zu diesen zählen neben beispielsweise Personalund Sachmitteln auch die liegenschaftsbezogenen Aufwendungen in Form sogenannter kalkulativer Kosten. Von den Bezirken muss im Budget eine angenommene Miete für die Nutzung einer bezirks- oder landeseigenen Liegenschaft ausgewiesen und auch gezahlt werden. Entscheidend ist, dass die kalkulierten Liegenschaftskosten mit einer bestimmten Verwaltungsleistung verbunden sind. Die Zuweisung dafür aus dem Landeshaushalt bemisst sich jedoch nicht nach den anfallenden Produktkosten eines Bezirks, sondern am Median der Kosten aller Berliner Bezirke. Für Liegenschaften, die nicht mit einem Produkt verbunden sind, müssen diese kalkulierten Mieten ebenso berechnet und gezahlt werden, wobei keine Gegenfinanzierung durch den Landeshaushalt existiert und in der Folge für diese Liegenschaften ein höherer Verwertungs- und Privatisierungsdruck entsteht.

Die Erfassung des öffentlichen Liegenschaftsbestands und die Produktion betriebswirtschaftlichen Wissens über die Nutzung von Liegenschaften führt über das Instrument der am Median der Produktkosten orientierten Budgetierung zu einer künstlichen Wettbewerbssituation zwischen den Bezirken. In einer Aufstellung zum intrabezirklichen Kostenvergleich heißt es dazu: „So entsteht Wettbewerb auch da, wo es Konkurrenz im eigentlichen Sinne nicht gibt.“ (Senatsverwaltung für Finanzen Berlin 2003: 3) Wie ein Fraktionsmitarbeiter des Berliner Abgeordnetenhauses darstellt, besteht eine weitere Vergleichsebene zwischen dem öffentlichen Liegenschaftsbestand und dem freien Immobilienmarkt: Das Budgetierungsverfahren „belastet meine amtliche Immobilie manchmal so, dass man sagen kann, wenn ich billig miete, komme ich günstiger." (B2012-E: 18) Darüber hinaus wurden die Bezirke anteilig an Verkaufserlösen beteiligt, was einen weiteren Anreiz zur ertragsorientierten Privatisierung öffentlicher Liegenschaften darstellt.

Die betriebswirtschaftliche Einhegung des öffentlichen Liegenschaftsbestands hat zu Folge, dass immer einige Bezirke eine strukturelle Unterfinanzierung aufweisen, der nur begegnet werden kann, wenn 
liegenschaftsbezogene Kosten reduziert beziehungsweise Liegenschaften verkauft werden. Die Liegenschaftsnutzung ist nicht an eine ausreichende Finanzierung der Bezirke geknüpft, sondern in erster Linie eine Frage des wirtschaftlichen Handelns der Bezirke selbst. In einem Bericht der Berliner Morgenpost wird der damalige Finanzsenator Peter Kurth (CDU) folgendermaßen zitiert: Die Bezirke „sollen direkt erfahren, dass sich Sparsamkeit und Einschränkung bei der Nutzung und Bewirtschaftung von Büroräumen lohnen. Genauso werden jene künftig Probleme haben, die verschwenderisch mit dieser Ressource umgehen." (Zit. n. Hintzmann 2001)

Die Wahrnehmung öffentlicher Liegenschaften unter den Bedingungen betriebswirtschaftlicher Verwaltungssteuerung ist des Weiteren von einer neuen Zeitlichkeit geprägt. Nicht die Gesamtnutzungsdauer einer Liegenschaft wird betrachtet, sondern die mit ihr verbundenen finanziellen Belastungen innerhalb eines Haushaltjahres, wie eine Berliner Bezirksrätin feststellt: „Diese Kurzfristigkeit im Haushalt, die Kurzfristigkeit in der politischen Verantwortungszeit deckt sich nicht mit dem, was man eigentlich nachhaltig braucht.“ (B2012-E: 64)

Die Gründung des Fonds als Privatisierungsmaschine und die betriebswirtschaftliche Einhegung der Liegenschaftsnutzung auf Ebene der Bezirke griffen dahingehend ineinander, dass das Portfolio des Fonds über Jahre mit Liegenschaften der Bezirke aufgefüllt wurde. Diese Konstellation geriet ab 2009 zunehmend in die Kritik[10], bis der Fonds schließlich 2015 als eigenständige Gesellschaft aufgelöst und mit einer anderen Gesellschaft des Landes Berlin zusammengelegt wurde. Dabei ist zu vermuten, dass der Fonds auch deswegen in seiner ursprünglichen Form abgewickelt wurde, da die Bezirke zur Kostenreduzierung immer mehr unattraktive Liegenschaften an den Fonds übertrugen und damit das Aktivierungspotential des zu vermarktenden Portfolios abnahm. In der Folge wurde es für den Fonds immer schwieriger, bedeutende Liegenschaftsverkäufe zu realisieren.

Der Liegenschaftsfonds steht für eine investoren- und aufwertungsorientierte Privatisierung öffentlichen Liegenschaftseigentums. Mit Hilfe der betriebswirtschaftlich geprägten Verwaltungsreform wurde das Handlungsfeld der Bezirke so strukturiert, dass ein Zugriff auf deren Liegenschaftsvermögen ermöglicht wurde, ohne diese direkt zu enteignen. Das kalkulative Regime handlungsleitender betriebswirtschaftlicher Wissensgenerierung ist nicht auf die Einführung einer neuen Verfahrensweise zu reduzieren, sondern als politisches Projekt einer unternehmerischen Stadtpolitik zu betrachten.

\section{Abschlussbetrachtung}

Die Betrachtung der beiden Fallbeispiele zeigt, dass die raumzeitlich und inhaltlich fragmentierte Umsetzung des NSM in den deutschen Städten und Kommunen mit „changes in the geometry of social power" (Swyngedouw 1997: 142) einhergeht, die in den Blick geraten, wenn die Verwaltungen nicht als mechanische Ausführungsorgane, sondern als strategisches Handlungsfeld betrachtet werden. Das Beispiel der Liegenschaftsverwaltung und -politik illustriert, dass unter den Bedingungen von Verwaltungsreform und Austeritätspolitik städtische und kommunale Verwaltungen zum zentralen Terrain zur Ausarbeitung und Artikulation einer unternehmerischen 
Stadtpolitik durch politische Eliten und privatwirtschaftliche Akteur_innen geworden sind. Abstrakter gesprochen handelt es sich bei der Verwaltungsreform um ein politisches Projekt der unternehmerischen Stadt: Mit ihr ändert sich „das konkrete Zusammenspiel von interessenbasierten rationalen Strategien, der Praktiken der diskursiven Interaktion sowie der Formen der affektiven Imagination“ (Bieling/Steinhilber 2000: 109). Das heißt, sie bildet das politisch-strategische Terrain, auf dem sich die unternehmerische Stadt verdichtet. Die Verwaltung der unternehmerischen Stadt ist die dialektische Einheit aus Voraussetzung und Ergebnis der inner- und außeradministrativen Artikulation einer unternehmerischen Politik auf städtischer Maßstabsebene, die kapitalintensive und aufwertungsorientierte Investitionen ermöglicht.

Die ökonomisierten administrativen Strukturen und Verfahrensweisen korrespondieren mit dieser dahingehend, dass Verwaltungshandeln entlang marktlicher und wettbewerblicher Verfahrensweisen und Handlungsrationalitäten begründet ist und auf lokaler Maßstabsebene strategische Schnittstellen und Handlungsfelder existieren, die privatwirtschaftlichen Akteur_innen einen privilegierten Zugang zu öffentlichen Vermögenswerten und Entscheidungsprozessen ermöglichen. In Frankfurt am Main wird die Rechtsform einer ÖPP vom damaligen Planungsdezernenten genutzt, um administrative Beteiligungsverfahren, politische Mitbestimmung und Fragen der Finanzierung zu umgehen sowie eine aufwertungs- und kapitalorientierte Stadtentwicklungspolitik für Haushalte mit mittleren und oberen Einkommen zu realisieren. In Berlin resultieren die Verschränkung inneradministrativer Reformprozesse und die Gründung einer privatrechtlichen Gesellschaft in der verwaltungsinternen Dominanz der Senatsverwaltung für Finanzen und der Inwertsetzung des öffentlichen Liegenschaftsbestands. Gerade die privatrechtlichen Gesellschaften ermöglichen es, Wissens-, Handlungs- und Finanzierungsressourcen projekt- und politikfeldbezogen jenseits traditioneller Finanzierungs- und Mitbestimmungsstrukturen zu erschließen. Sie fungieren als formalisierte und ausschließende Interfaces für die Synchronisierung öffentlicher und privatwirtschaftlicher Interessen. Entscheidungen werden in enger Abstimmung und Kooperation mit privaten Investor_innen außerhalb der traditionellen Entscheidungs- und Verwaltungsstrukturen ausgearbeitet, beschlossen und umgesetzt.

Sowohl die Mobilisierung der Verwaltungsreform als auch die reformierte Verwaltungspraxis an sich konstituieren eine handlungsleitende Wissensordnung. Die in die Reformen eingeschriebene Problematisierung und Messung betriebswirtschaftlicher Effektivität und Effizienz ist kein deskriptives Wissen, sondern ein konstruierender und handlungsleitender Macht/Wissen-Komplex. Die damit verbundene Sinnmachung strukturiert das Handlungsfeld von Politiker_innen und Verwaltungsmitarbeiter_innen und ist dahingehend handlungsleitend, dass administratives und politisches Handeln sich immer (auch) über Wirtschaftlichkeitskriterien legitimieren müssen, die Objektivität, Transparenz und Gerechtigkeit suggerieren. Demgegenüber treten politische Ziele und partikulare Interessen in den Hintergrund. Im Kontext öffentlicher Liegenschaftspolitik und aktueller Konflikte um bezahlbaren Wohnraum, fortschreitende Verdrängung, die Unterbringung von Geflüchteten oder soziale, politische und kulturelle 
Initiativen zeigt sich jedoch, dass die Beantwortung der Frage, wie gerade in hochverdichteten urbanen Räumen mit Grund und Boden umgegangen wird, nicht einer marktförmigen Regulation überlassen werden kann.

\section{Gefördert durch Mittel des Open-Access-Publikationsfonds der Goethe Universität Frankfurt.}

\section{Endnoten}

[1] Die Ausführungen basieren auf meiner Dissertationsschrift, die im April 2018 unter dem Titel Die Verwaltung der unternehmerischen Stadt. Zur neoliberalen Neuordnung von Liegenschaftspolitik und-verwaltung in Berlin und Frankfurt am Main im transcript Verlag (Bielefeld) erschienen ist.

[2] Rick Vogel (2006: 337) unterscheidet hierbei vier Phasen des Reformprozesses. In der Geburtsphase (1989-1991) wird ein gemeinsames Problembewusstsein formuliert. In der Wachstumsphase (1991-1995) findet eine offensive Propagierung und Mobilisierung des NSM durch die KGSt statt, wobei der Fokus auf der Binnenreform liegt. In der Reifephase (1995-1999) tritt neben der Reform der Binnenstruktur ein rechtswissenschaftlich geprägter Privatisierungsdiskurs. Die Verfallsphase (1999-2003) ist von einer Dominanz des Privatisierungsdiskurses gegenüber dem NSM-Diskurs geprägt.

[3] Nach zehn Jahren Reformprozess geben 92,4 Prozent der befragten Kommunen ( $\mathrm{n}=840)$ in einer von der Hans-Böckler-Stiftung und der KGSt geförderten Studie (Bogumil/ Grohs/Kuhlmann 2006: 7-8, siehe auch Jann et al. 2004, Bogumil et al. 2007) an, Reformmaßnahmen ergriffen zu haben. 82,4 Prozent haben sich dabei am NSM als Leitbild für die Reformen orientiert, wobei 66,3 Prozent der Kommunen nur einzelne Instrumente umgesetzt haben. Lediglich 2,5 Prozent der befragten Kommunen haben eine umfassende Umsetzung des NSM erreicht.

[4] Im Diskurs der Verwaltungsreform wird Liegenschaftsverwaltung ausschließlich als Liegenschaftsbewirtschaftung und Facility Management thematisiert (vgl. Kommunale Gemeinschaftsstelle für Verwaltungsvereinfachung 1991; 1993a).

[5] Eindrücklich diskutiert Nils Schmid (2005) in seiner staatsrechtlich geprägten Arbeit den Zusammenhang zwischen Staatssouveränität und Staatsvermögen. Er stellt fest, dass zwar keine eindeutige Bemessung einer verfassungsmäßigen Eigentumsquote möglich ist, sich aber „mit der Verfügung über Staatsvermögen [...] auch das Ausmaß an eigenständiger Gestaltungsmacht und Planungsmöglichkeiten“ (197) der öffentlichen Hand entscheidet.

[6] Die Interviews wurden im Rahmen des von der DFG geförderten Projekts „Neuordnung städtischer Verwaltungen: New Public Management am Beispiel der Liegenschaftsverwaltungen in Berlin und Frankfurt am Main“ geführt. Zu den beiden Fallbeispielen Frankfurt am Main und Berlin wurden insgesamt 29 leitfadengestützte Expert_inneninterviews geführt. Interviewt wurden aktuelle und ehemalige Repräsentant_innen aus Verwaltung, Politik, (öffentlichen) Unternehmen und Zivilgesellschaft, die über relevantes Deutungs- und Verfahrenswissen hinsichtlich des Forschungsinteresses verfügen.

[7] In Frankfurt am Main wird ab Mitte der 1990er Jahre eine Reform der Binnenadministration begonnen, deren Erfolg als sehr beschränkt bewertet wird. Dies wird unter anderem damit begründet, dass erstens der Handlungsdruck nie besonders groß war, zweitens verwaltungsinterne Widerstände den Reformprozess behinderten und drittens die hohe politische Autonomie der einzelnen Magistratsmitglieder zu einer Vielzahl inkompatibler Insellösungen geführt hat.

[8] Der Berliner Verwaltungsapparat besteht aus den Bezirks- und den Senatsverwaltungen. Den Bezirken kommt dabei die Aufgabe zu - wie eine Kommune - die tatsächliche Leistungserbringung zu gewährleisten. Die Bezirke verfügen über eine hohe Fachautonomie, sind aber finanziell vom Senat und dem Berliner Abgeordnetenhaus abhängig, da das Budget der Bezirke Teil des Berliner Landeshaushaltes ist. 
[9] Mehr noch kann davon gesprochen werden, dass das Liegenschaftsvermögen überhaupt erst sichtbar gemacht wurde. Bis zur Gründung des Fonds existierte keine systematische und umfassende Übersicht über das bezirks- und landeseigene Liegenschaftsvermögen.

[10] Eine erste deutliche Kritik wurde im Jahr 2009 auf dem Stadtforum Berlin geäußert, das von der Senatsverwaltung für Stadtentwicklung organisiert wurde (vgl. SenStadt Berlin 2009). Die damalige Senatorin für Stadtentwicklung, Ingeborg Junge-Reyer, fordert in ihrem Statement eine gestaltende Liegenschaftspolitik als Instrument zur Realisierung wirtschafts-, stadtentwicklungs-, wohnungs- und sozialpolitischer Zielsetzungen. Im Jahr 2011 wurde die stadtpolitische ,Initiative Stadt Neudenken“ von zivilgesellschaftlichen Aktiven aus den Bereichen Kultur, Wissenschaft, Architektur gegründet. Sie hat wesentlich dazu beigetragen, die Berliner Liegenschaftspolitik auf die politische Agenda zu setzen (vgl. Vollmer 2015: 59-6o). Auf Druck der Initiative wurde gemeinsam mit den Fraktionen im Berliner Abgeordnetenheus 2012 der ,Runde Tisch zur Neuausrichtung der Berliner Liegenschaftspolitik' gegründet, der wesentlich zur Reform der Berliner Liegenschaftspolitik beigetragen hat und bis zum gegenwärtigen Zeitpunkt den Umgang mit Berliner Liegenschaftsvermögen kritisch begleitet (kritisch zur Neuausrichtung der Berliner Liegenschaftspolitik Silomon-Pflug 2018: 201-206).

\section{Autor innen}

Felix Silomon-Pflug ist Politologe und Geograph. Er arbeitet zu Themen Stadtgeographie, Urban Governance, Geographien des Landbesitzes und Labour Geography. silomon@geo.uni-frankfurt.de

\section{Literatur}

Adamaschek, Bernd (1997): Interkommunaler Leistungsvergleich. Leistung und Innovation durch Wettbewerb. Gütersloh: Verlag Bertelsmann-Stiftung.

Adolphs, Stephan (2008): Geschichte der Gouvernementalität oder materialistische Staatstheorie? Michel Foucault und Nicos Poulantzas im Vergleich. In: Joachim Hirsch / John Kannankulam / Jens Wissel (Hg.), Der Staat der Bürgerlichen Gesellschaft. Zum Staatsverständnis von Karl Marx. Baden-Baden: Nomos, 180-202.

Andresen, Sünne / Dölling, Irene / Kimmerle, Christoph (Hg.) (2003): Verwaltungsmodernisierung als soziale Praxis. Geschlechter-Wissen und Organisationsverständnis von Reformakteuren. Wiesbaden: VS Verlag.

Banner, Gerhard (1991): Von der Behörde zum Dienstleistungsunternehmen: Die Kommunen brauchen ein neues Steuerungsmodell. In: Verwaltungsführung, Organisation, Personal 13, 6-11.

Banner, Gerhard (1994): Anregungen für die deutsche Verwaltungsreform. In: Carl Bertelsmann-Preis 1993. Demokratie und Effizienz in der Kommunalverwaltung. Gütersloh: Verlag Bertelsmann-Stiftung, 349-364.

Belina, Bernd / Dzudzek, Iris (2009): Diskursanalyse als Gesellschaftsanalyse. Ideologiekritik und Kritische Diskursanalyse. In: Georg Glasze / Annika Mattissek (Hg.), Handbuch Diskurs und Raum. Theorien und Methoden für die Humangeographie sowie die sozialund kulturwissenschaftliche Raumforschung. Bielefeld: transcript, 129-152.

Bertelsmann Stiftung (1994a): Carl Bertelsmann-Preis 1993. Demokratie und Effizienz in der Kommunalverwaltung. Gütersloh: Verlag Bertelsmann-Stiftung.

Hintzmann, Karsten (2001): Berlin zahlt Millionen für leere Büros. Finanzsenator Kurth will mit Gebäudemanagement Geld sparen, in: Berliner Morgenpost, 15.1.2001, 25.

Bieling, Hans-Jürgen / Steinhilber, Jochen (2000): Hegemoniale Projekte im Prozess der europäischen Integration. In: Hans-Jürgen Bieling / Jochen Steinhilber (Hg.): Die Konfiguration Europas. Dimensionen einer kritischen Integrationstheorie. Münster: Westfälisches Dampfboot, 102-130.

Blomley, Nicolas (2003): Unsettling the city. Urban land and the politics of property. London: Routledge. 
Bogumil, Jörg / Grohs, Stephan / Kuhlmann, Sabine (2006): Ergebnisse und Wirkungen kommunaler Verwaltungsmodernisierung in Deutschland. Eine Evaluation nach zehn Jahren Praxiserfahrung. In: Jörg Bogumil / Werner Jann / Frank Nullmeier (Hg.), Politik und Verwaltung. Wiesbaden: VS Verlag, 151-184.

Bogumil, Jörg / Grohs, Stephan / Kuhlmann, Sabine / Ohm, Anna K. (2007): Zehn Jahre Neues Steuerungsmodell. Eine Bilanz kommunaler Verwaltungsmodernisierung. Berlin: Edition Sigma.

Brenner, Neil (2004): New State Spaces. Urban Governance and the Rescaling of Statehood. Oxford: Oxford University Press.

Brenner, Neil / Theodore, Nik (2002): Cities and the geographies of "actually existing neoliberalism". In: Antipode 34/3, 349-379.

Budäus, Dietrich / Hilgers, Dennis (2010): Reform kommunaler Verwaltungen in Deutschland - Entwicklung, Schwerpunkte und Perspektiven. In: Wolfgang Schuster / Klaus-Peter Murawski (Hg.), Die regierbare Stadt. Stuttgart: Kohlhammer/Deutscher Gemeindeverlag, 80-107.

Carl Bertelsmann-Preis 1993. Demokratie und Effizienz in der Kommunalverwaltung (1994b). Gütersloh: Verlag Bertelsmann-Stiftung.

Damkowski, Wulf / Precht, Claus (1998): Public Management in Deutschland: Neuere Entwicklungen und eine Zwischenbilanz. In: Wulf Damkowski / Claus Precht (Hg.), Moderne Verwaltung in Deutschland: Public-Management in der Praxis. Stuttgart: Kohlhammer, 15-33.

Demirović, Alex (2007a [1987]): Nicos Poulantzas. Aktualität und Probleme materialistischer Staatstheorie. Münster: Westfälisches Dampfboot.

Demirović, Alex (2007b): Politische Beratung, Think Tanks und Demokratie. In: Jens Wernicke / Torsten Bultmann (Hg.), Netzwerk der Macht - Bertelsmann. Der medial-politische Komplex aus Gütersloh. Marburg: BdWi-Verlag, 15-29.

Demirović, Alex (2011): Governance - eine neue Stufe staatlicher Herrschaft. In: Alex Demirović / Heike Walk (Hg.), Demokratie und Governance. Kritische Perspektiven auf neue Formen politischer Herrschaft. Münster: Westfälisches Dampfboot, 73-105.

Deutscher Beamtenbund und Tarifunion (1996): DBB-Konzept zur Dienstrechts- und Verwaltungsreform. Bonn.

Diaz-Bone, Rainer (2006): Die interpretative Analytik als methodologische Position. In: Brigitte Kerchner / Silke Schneider (Hg.), Foucault: Diskursanalyse der Politik. Eine Einführung. Wiesbaden: VS Verlag, 68-84.

Esser, Josef / Hirsch, Joachim (1982): Materialistische Staatstheorie und Verwaltungswissenschaft. In: Joachim Jens Hesse (Hg.), Politikwissenschaft und Verwaltungswissenschaft. Opladen, 105-121.

Felder, Michael (1998): Vom „muddling through“ zurück zum „eisernen Käfig“? Aktuelle Strategien der Verwaltungsmodernisierung. In: Zeitschrift Marxistische Erneuerung 34, 91-109.

Felder, Michael (2000): Verwaltungsmodernisierung, die Transformation von Staatlichkeit und die neue Sozialdemokratie. In: UTOPIE kreativ 121/122, 1090-1102.

Felder, Michael (2001): Die Transformation von Staatlichkeit. Europäisierung und Bürokratisierung in der Organisationsgesellschaft. Wiesbaden: Westdeutscher Verlag.

Foucault, Michel (1977): Überwachen und Strafen. Die Geburt des Gefängnisses. Frankfurt am Main: Suhrkamp.

Foucault, Michel (1981 [1973]): Archäologie des Wissens. Frankfurt am Main: Suhrkamp.

Fuller, Crispian (2010): Crisis and Institutional Change in Urban Governance. In: Environment and Planning A 42/5, 1121-1137.

Goodwin, Mark / Painter, Joe (1996): Local Governance, the Crises of Fordism and the Changing Geographies of Regulation. In: Transactions of the Institute of British Geographers $21 / 4,635-648$.

Gottweis, Herbert (2004): Theoretical Strategies of Poststructuralist Policy Analysis: Towards an Analytics of Government. In: Maarten A. Hajer / Hendrik Wagenaar (Hg.), Deliberative Policy Analysis. Understanding Governance in the Network Society. Cambridge: Cambridge University Press, 247-265.

Harvey, David (1989): From Managerialism to Entrepreneurialism: The Transformation in Urban Governance in Late Capitalism. In: Geografiska Annaler 71/1, 3-17.

Heeg, Susanne (2008): Von Stadtplanung und Immobilienwirtschaft. Die „South Boston Waterfront“ als Beispiel für eine neue Strategie städtischer Baupolitik. Bielefeld: transcript. 
Jann, Werner / Bogumil, Jörg / Bouckaert, Geert / Budäus, Dietrich / Holtkamp, Lars / Kißler, Leo / Kuhlmann, Sabine / Mezger, Erika / Reichard, Christoph / Wollmann, Hellmut (Hg.) (2004): Status-Report Verwaltungsreform. Eine Zwischenbilanz nach zehn Jahren. Berlin: Edition Sigma.

Jessop, Bob (1992): Regulation und Politik. Integrale Ökonomie und integraler Staat. In: Alex Demirović / Hans-Peter Krebs / Thomas Sablowski (Hg.), Hegemonie und Staat. Kapitalistische Regulation als Projekt und Prozeß. Münster: Westfälisches Dampfboot, 232-262.

Jessop, Bob (2010): Cultural Political Economy and Critical Policy Studies. In: Critical Policy Studies 3/3-4, S. 336-356.

Koch, Rainer (2004): Umbau Öffentlicher Dienste. Internationale Trends in der Anpassung Öffentlicher Dienste an ein New Public Management. Wiesbaden: Deutscher Universitäts-Verlag.

Koenigs, Tom / Wentz, Martin (1994): Gründung der KEG Konversions-Entwicklungsgesellschaft, M 205 v. 14.10.1994. Frankfurt am Main.

Kommunale Gemeinschaftsstelle für Verwaltungsvereinfachung (1991): Mobilisierung von Grundstücken für investive Zwecke durch die Gemeinden und Kreise in den neuen Bundesländern. Köln.

Kommunale Gemeinschaftsstelle für Verwaltungsvereinfachung (1992): Wege zum Dienstleistungsunternehmen Kommunalverwaltung. Fallstudie Tilburg. Köln.

Kommunale Gemeinschaftsstelle für Verwaltungsvereinfachung (1993a): Organisationsmodell für Gemeinden der GK 5. Organisation des Wirtschaftsförderungs- und Liegenschaftsamtes. Köln.

Kommunale Gemeinschaftsstelle für Verwaltungsvereinfachung (1993b): Das Neue Steuerungsmodell. Begründung, Konturen, Umsetzung. Köln.

Lebuhn, Henrik (2007): Sozialräumliche Konflikte in der unternehmerischen Stadt. Eine Berliner Fallstudie über städtische Bewegungspolitik unter den Bedingungen von New Public Manaqement. In: PROKLA 37/149, 529-545.

Lebuhn, Henrik (2008): Stadt in Bewegung. Mikrokonflikte um den öffentlichen Raum in Berlin und Los Angeles. Münster: Westfälisches Dampfboot.

Lebuhn, Henrik (2010): Das Neue Steuerungsmodell und die (Markt-)Logik städtischer Verwaltungen. In: Zeitschrift Marxistische Erneuerung 21/83, 35-45.

Liegenschaftsfonds Berlin (2014): Mein Zuhause vom Land Berlin. Objekte im Bieterverfahren Mai 2014. Berlin.

McCann, Eugene / Ward, Kevin (2011): Urban assemblages. Territories, relations, practices, and power. In: Eugene McCann / Kevin Ward (Hg.), Mobile Urbanism. Cities and Policymaking in the Global Age. Minneapolis: University of Minnesota Press, xiii-xxxv.

Naschold, Frieder (1995): Ergebnissteuerung, Wettbewerb, Qualitätspolitik. Entwicklungspfade des öffentlichen Sektors in Europa. Berlin: Edition Sigma.

Ong, Aihwa (2006): Neoliberalism as a Mobile Technology. In: Transactions of the Institute of British Geographers 32/1, 3-8.

Peck, Jamie (2010): Constructions of Neoliberal Reason. Oxford, New York: University Press.

Pelizzari, Alessandro (2001): Die Ökonomisierung des Politischen. New Public Management und der neoliberale Angriff auf die öffentlichen Dienste. Konstanz: UVK.

Pelizzari, Alessandro (2004): Entpolitisiertes Verwaltungswissen. Ökonomisierungstendenzen am Beispiel des Zürcher Spitalwesens. In: Peter Collin / Thomas Horstmann (Hg.), Das Wissen des Staates. Geschichte, Theorie und Praxis. Baden-Baden: Nomos, 434-457.

Polanyi, Karl (2001 [1944]): The Great Transformation. The Political and Economic Origins of Our Time. Boston, Massachusetts: Beacon Press.

Poulantzas, Nicos (2002 [1978]): Staatstheorie. Politischer Überbau, Ideologie, autoritärer Etatismus. Hamburg: VSA.

Robinson, Jennifer (2011): The spaces of circulating knowledge. In: Eugene McCann / Kevin Ward (Hg.), Mobile Urbanism. Cities and Policymaking in the Global Age. Minneapolis: University of Minnesota Press, 15-40.

Schipper, Sebastian (2018): Wohnraum dem Markt entziehen? Wohnungspolitik und städtische soziale Bewegungen in Frankfurt und Tel Aviv. Wiesbaden: Springer VS.

Schmid, Nils (2005): Staatliches Liegenschaftsmanagement, Staatsverschuldung und Staatsvermögen. Berlin: Duncker \& Humblot. 
Senatsverwaltung für Finanzen Berlin (2003): Was kostet wo wie viel? Berliner Bezirke im Kostenvergleich. Berlin.

Silomon-Pflug, Felix (2018): Die Verwaltung der unternehmerischen Stadt. Zur neoliberalen Neuordnung von Liegenschaftspolitik und -verwaltung in Berlin und Frankfurt am Main. Bielefeld: transcript.

SenStadt Berlin - Senatsverwaltung für Stadtentwicklung Berlin (2009): Stadtforum Berlin. „Alles muss raus? Die Zukunft der Liegenschaftspolitik in Berlin“. Dokumentation der Veranstaltung. Berlin. Online verfügbar unter https://digital.zlb.de/viewer/ rest/image/33655284/alles_raus.pdf/full/max/o/alles_raus.pdf (letzter Zugriff am 21.06.2018).

Swyngedouw, Erik (1997): Neither global nor local. „Glocalization“ and the politics of scale. In: Kevin R. Cox (Hg.), Spaces of Globalization. Reasserting the Power of the Local. New York: Guilford Press, 137-166.

Vogel, Rick (2006): Zur Institutionalisierung von New Public Management. Disziplindynamik der Verwaltungswissenschaft unter dem Einfluss ökonomischer Theorie. Wiesbaden: Deutscher Universitäts-Verlag.

Vollmer, Lisa (2015): Die Berliner Mieter_innenbewegung zwischen lokalen Konflikten und globalen Widersprüchen. In: Sozial.Geschichte Online (17), S. 51-82.

Wentz, Martin (1991): Raum und Zeit in der metropolitanen Entwicklung. In: Martin Wentz (Hg.), Stadt-Räume. Frankfurt am Main/New York: Campus, 9-14.

\section{Administration of the Entrepreneurial City. (No) Topic for geographical urban research?!}

In geographical urban research there are general references to the fact that the canon of neoliberal rescaling and urban transformation also includes the introduction of New Public Management in the cities of western industrial nations. Subsequently, I argue that what I summarize as the administration of the entrepreneurial city is not merely the result of abstract neoliberalisation processes or technocratic modernization of a mechanical executive apparatus. In this article I show that the economically reformed administration is both effect of and important terrain for the development, articulation and implementation of an entrepreneurial urban policy. Therefore, I propose to understand the research object urban administration as a strategic field, which is the result and terrain of social power relations. Furthermore, I understand the process of administrative reform as a power/knowledge complex in order to show how an entrepreneurial understanding of local government action can be established with the help of problematisation and knowledge regimes. Using the example of effects of administrative reforms on the perception and handling of public real estate assets in Frankfurt am Main and Berlin since the 199oies, I will show how internal administrative structures were economized and private legal forms were (and still are) used to pursue valorisation-oriented strategies in the context of an entrepreneurial urban policy through the dynamisation and flexibilisation of administrative apparatus. 